Service social

\title{
Expérience des familles dont un enfant présente une incapacité : perceptions et besoins actuels
}

\section{Carmen Dionne, Nadia Rousseau, Caroline Drouin, Caroline Vézina et Suzie McKinnon}

Volume 52, numéro 1, 2006

URI : https://id.erudit.org/iderudit/015955ar

DOI : https://doi.org/10.7202/015955ar

Aller au sommaire du numéro

Éditeur(s)

École de service social de l'Université Laval

ISSN

1708-1734 (numérique)

Découvrir la revue

Citer cet article

Dionne, C., Rousseau, N., Drouin, C., Vézina, C. \& McKinnon, S. (2006).

Expérience des familles dont un enfant présente une incapacité : perceptions et besoins actuels. Service social, 52(1), 65-77. https://doi.org/10.7202/015955ar

\section{Résumé de l'article}

La présence d'une incapacité chez un enfant peut poser des défis importants à sa famille. Cet article traite de perceptions des parents concernant cette expérience ainsi que leurs besoins. Il s'agit d'une recherche de type qualitatif. Des entrevues individuelles semi-dirigées ont été réalisées auprès de 24 parents d'enfants présentant les incapacités suivantes : 1) incapacités intellectuelles, 2) problèmes de santé mentale, 3) incapacités visuelles, 4) incapacités auditives, 5) incapacités motrices et 6) traumatisme craniocérébral. Les entrevues ont été analysées à l'aide du logiciel Atlas.ti (Muhr, 1997). Les résultats traitent de leur appréciation des aides et services reçus et de leur expérience familiale. 


\section{Expérience des familles dont un enfant présente une incapacité : perceptions et besoins actuels}

Carmen DionNE

Professeure

Département de psychoéducation

Université du Québec à Trois-Rivières

Nadia RousSEAU

Professeure

Département des sciences de l'éducation

Université du Québec à Trois-Rivières

Caroline DROUIN

Professionnelle de recherche

Département de psychoéducation

Université du Québec à Trois-Rivières

Caroline VÉZINA

Assistante étudiante

Département des sciences de l'éducation

Université du Québec à Trois-Rivières

Suzie McKinNon

Professionnelle de recherche Département de psychoéducation

Université du Québec à Trois-Rivières

La présence d'une incapacité chez un enfant peut poser des défis importants à sa famille. Cet article traite de perceptions des parents concernant cette expérience ainsi que leurs besoins. II s'agit d'une recherche de type qualitatif. Des entrevues individuelles semi-dirigées ont été réalisées auprès de 24 parents d'enfants présentant les incapacités suivantes : 1) incapacités intellectuelles, 2) problèmes de santé mentale, 
3) incapacités visuelles, 4) incapacités auditives, 5) incapacités motrices et 6) traumatisme craniocérébral. Les entrevues ont été analysées à l'aide du logiciel Atlas.ti (Muhr, 1997). Les résultats traitent de leur appréciation des aides et services reçus et de leur expérience familiale.

\section{Mots-clés : besoins des parents, incapacités, services, enfants.}

The presence of a child with a disability can pose important challenges within the family. This article concerns the parents' perceptions of their life experience and their needs. This qualitative design research was realized with 24 parents of children with the following incapacities: 1) intellectual disabilities, 2) mental health problems, 3) visual impairment, 4) hearing impairment, 5) physical disabilities, and 6) traumatic brain injury. The semi-directed individual interviews were analyzed with the software ATLAS.ti (Muhr, 1997). The results present informations about their appreciation of the assistances and services they received and their family life experience.

\section{Keywords: Parents' needs, Disabilities, Services, Children.}

\section{INTRODUCTION}

Au Québec, il existe environ 116400 enfants dont l'âge se situe entre la naissance et 14 ans et qui présentent une incapacité (Camirand, 2001). Non seulement la présence de cette incapacité perturbe le développement de ces jeunes, mais elle touche également leur famille. Les impacts peuvent être nombreux. L'aspect financier compte parmi les impacts importants, que ce soit par les coûts directs (ex. : régimes alimentaires particuliers, transport, gardiennage, médication, équipement spécialisé et aide technique, etc.) ou par les coûts indirects (ex. : épuisement parental, ruptures familiales, absentéisme au travail) (Camirand et Aubin, 2004; L'Institut Roeher, 2000). Soulignons que ces coûts indirects, quoique tout aussi importants, peuvent être plus difficilement évaluables et qu'ils peuvent toucher différentes dimensions de la vie des familles.

Chez les parents dont un enfant présente une incapacité, des modifications relatives à l'exercice des rôles parentaux sont observées. Les parents sont en effet appelés à jouer une variété de rôles auprès de l'enfant. L'utilisation des services spécialisés, pourtant destinés à soutenir le rôle parental, peut être source de multiples insatisfactions: manque de disponibilité du personnel, programmes dont le financement est limité, services fragmentés et non concertés, et accès difficile aux programmes, notamment au répit et à l'intégration des enfants en centres de la petite enfance (voir entre autres: Darling, 1991; Gazzoni, 1998; Perreault, 1997; Tougas, 2002). Cette expérience de rôles variés n'est pas sans conséquences pour le parent. Guralnick (2000) voit la menace au sentiment de compétence des parents comme une répercussion possible de la présence d'une incapacité chez l'enfant. Des effets psychologiques chez les familles de la présence de l'incapacité ont également été documentés. Le climat familial et les relations entre les conjoints peuvent aussi être affectés sur le plan des 
relations familiales. Par exemple, la possibilité d'une rupture familiale, d'un refus d'avoir d'autres enfants, une moins grande énergie et une disponibilité réduite pour les activités de couple, moins de moments pour l'intimité ainsi que les possibilités limitées de reconstitution de nouveaux couples sont parmi les nombreux impacts rapportés dans les écrits scientifiques (Crnic, Friedrich et Greenberg, 1983; Dyson, 1993; Tétreault, 1993, 2002).

Comme les parents, la fratrie est touchée par la présence d'une incapacité chez l'un des enfants. Des recherches font d'ailleurs état des effets positifs et négatifs sur les autres enfants. Parmi les effets négatifs, Scelles (1994) mentionne les problèmes psychologiques, les problèmes d'identité face à l'enfant handicapé ainsi que les problèmes liés à l'organisation des rôles et des tâches dans la famille. De plus, selon Tétreault (1993), un appauvrissement du réseau social, des difficultés de relations avec l'entourage, de même qu'une diminution des activités sociales et de loisirs chez les parents d'enfants ayant des incapacités, contribueraient à un sentiment d'isolement social. Les parents ont également tendance à attribuer leur stress et leur fatigue au manque de soutien plutôt qu'au handicap de leur enfant (L'Institut Roeher, 2000). Les impacts de l'incapacité ne se limitent pas uniquement à la famille immédiate de l'enfant. Les grands-parents peuvent également être touchés par cette situation tant sur le plan affectif que dans leurs besoins d'information (Gagnier et Gosselin, 1999).

Cet article présente les résultats d'une étude financée par l'Office des personnes handicapées du Québec (OPHQ) et réalisée par Dionne et Rousseau (2006), dont l'objectif principal était de déterminer les dimensions de la vie familiale pour lesquelles une intervention gouvernementale était nécessaire en vue d'améliorer la condition de vie de ces familles et de leur entourage. La situation des familles québécoises comptant un enfant ayant une incapacité est-elle similaire à celle qui est rapportée dans les écrits scientifiques américains?

Six types d'incapacités, mutuellement exclusives pour la majorité des participants, sont couvertes par cette étude : 1) les incapacités intellectuelles, 2) les problèmes de santé mentale, 3) les incapacités visuelles, 4) les incapacités auditives, 5) les incapacités motrices et 6) le traumatisme craniocérébral. Cette recherche vise à rendre compte de la perspective des parents afin de mieux comprendre leur situation de vie.

\section{MÉTHODE}

Ce projet de recherche s'inscrit dans un paradigme qualitatif qui se rapproche de l'étude de cas et, plus spécifiquement, de l'étude de cas propre à une situation (situation analysis) (Bogdan et Biklen, 1998). Dans le présent article, il s'agit d'une situation familiale où vit un enfant ayant une incapacité. Conformément à la méthode utilisée dans ce type d'études de cas, le processus de collecte de données est passé d'un mode général à un mode spécifique. En effet, on a d'abord réuni deux groupes de discussion (focus groups) formés d'un intervenant et de sept parents d'enfants ayant 
des incapacités (un groupe était composé de quatre parents d'enfants présentant des incapacités intellectuelles, des troubles envahissants de développement et de la dysphasie, alors que le deuxième groupe comprenait une intervenante et trois parents d'enfants ayant une déficience physique). L'objectif des réunions de groupe était de soutenir la construction de la grille d'entrevue utilisée pour les rencontres individuelles ultérieures. Les participants ont été interrogés sur les impacts de l'incapacité dans la vie quotidienne et sur les parcours de vie (choix de vie), ainsi que sur l'utilisation du réseau naturel et du réseau formel (services). Les dimensions importantes retenues par les familles elles-mêmes ont été utilisées dans l'élaboration du protocole d'entrevue. Ce dernier a ensuite fait l'objet d'une présentation au comité de recherche de l'OPHQ. Quelques modifications ont été apportées au protocole à la suite des recommandations du comité de la recherche.

\section{ENTREVUES INDIVIDUELLES}

Par ailleurs, des entrevues individuelles semi-dirigées ont été menées auprès de 24 parents d'enfants ayant des incapacités, soit quatre parents par type d'incapacité. La participation de ces familles était volontaire. Notons, toutefois, qu'on a retenu en priorité les participants appartenant à des milieux variés (milieu rural ou urbain, famille monoparentale ou biparentale, un ou deux enfants ou plus de deux enfants, avec statut socioéconomique [SSÉ] faible ou élevé, avec une incapacité légère ou grave), en plus de présenter des caractéristiques différentes (utilisation du braille, de la langue des signes québécoise, de l'oralisme ou d'une aide technique adaptée, variation de l'âge et du nombre d'enfants ayant une incapacité). Les participants ont été recrutés par l'entremise d'organismes de promotion des personnes handicapées et des milieux de pratique. La consigne à respecter par ces organismes était de proposer des familles aux caractéristiques variées, selon la description donnée précédemment. En fonction de la disponibilité des participants, ces caractéristiques ont été considérées dans la formation de l'échantillon.

Quatre des 24 entrevues se sont déroulées en présence des deux parents, trois rencontres ont eu lieu avec le père seulement et dix-sept, avec la mère seulement. Toutes les entrevues ont été réalisées selon la disponibilité des parents et leur situation familiale. Douze familles venaient d'un milieu rural et les douze autres appartenaient à un milieu urbain. L'échantillon comprenait également neuf familles monoparentales et quinze familles biparentales. Le SSÉ est élevé pour cinq familles, moyen pour quinze autres familles et faible pour trois d'entre elles. Les entrevues individuelles se sont déroulées à la résidence du participant entre avril et décembre 2004. Elles étaient d'une durée moyenne de 45 minutes, bien qu'aucune limite de temps n'ait été prévue.

\section{ANALYSE DES DONNÉES}

Les données des entrevues individuelles sont enregistrées sur support audio pour ensuite être transcrites intégralement et analysées à l'aide du logiciel Atlas.ti (Muhr, 
1997). Les verbatims sont codés et caractérisés selon leur parenté ou leur similitude, sans qu'il y ait de code prédéterminé au départ (L'Écuyer, 1987). Le traitement des données a pour objectif de faire ressortir les significations de chacun des éléments de façon inductive. S'inspirant du codage par réseau (Cohen, Manion et Morrison, 2000), l'ensemble des données analysées donne lieu à l'élaboration d'un système de catégories grâce à la classification des données. Ce mode d'analyse permet d'organiser et d'illustrer les liens existant entre les différentes catégories de données sans en perdre la subtilité ou la complexité.

\section{RÉSULTATS}

L'analyse des verbatims permet l'identification de 3957 unités de sens. Quatre thèmes distincts émergent de l'analyse et sont présentés en fonction de leur importance relative, ainsi qu'en témoigne le tableau ci-dessous. Toutefois, seuls les deux premiers thèmes sont traités dans cet article : l'appréciation des aides et services et l'expérience familiale au quotidien (implication des parents et adaptation de la vie familiale, attitudes des autres à l'égard de la situation, difficultés dans la vie familiale).

Distribution des 3957 unités de sens dans les thèmes retenus à la suite des entrevues réalisées auprès des parents d'enfants ayant des incapacités

Thèmes

\begin{tabular}{lrr}
\hline Appréciation des aides et services & 1658 & $(42 \%)$ \\
Expérience familiale au quotidien & 1165 & $(29 \%)$ \\
Situation de l'enfant & 593 & $(15 \%)$ \\
Intégration scolaire de l'enfant & 541 & $(14 \%)$ \\
Total & $\mathbf{3 9 5 7}$ & $\mathbf{( 1 0 0 \% )}$
\end{tabular}

\section{Thème : Appréciation des aides et services}

Le thème «Appréciation des aides et services » regroupe les catégories suivantes: les améliorations de l'aide et des services, l'inventaire de l'aide et des services reçus, les aides et services perçus positivement, les informations requises et les coûts associés à l'incapacité.

Comme son nom l'indique, cette catégorie regroupe tous les commentaires négatifs que les parents ont formulés au sujet de l'aide et des services qu'ils ont reçus ou qu'ils auraient aimé recevoir. L'élément qui cause le plus d'insatisfaction est le délai d'attente (pour recevoir des services et pour avoir accès aux spécialistes). Par ailleurs, les parents soulignent que les spécialistes (médecin spécialiste, ergothérapeute, physiothérapeute, orthophoniste, etc.) ne seraient pas assez nombreux pour répondre à la 
demande. Ils font aussi remarquer que les enfants doivent sans cesse consulter de nouveaux intervenants et s'y habituer :

Il y a souvent trop de changements d'intervenants aussi, [...] il y a une madame qui est arrivée et elle savait de quoi elle parlait, elle a cerné tout de suite [mon enfant], elle l'a vu tout de suite, tout de suite, tout de suite. [...] Après ça, elle est partie en vacances, ils ont passé sur une autre intervenante qui, elle, ne connaissait pas le dossier du tout, je pense qu'elle ne s'était même pas donné la peine de lire le dossier avant de nous rencontrer. $(2: 2339)$

Les associations seraient souvent les premières à offrir du soutien aux parents, devançant les services de santé :

En tout cas, l'Association, c'est très important pour nous aider, ça va tout chercher les ressources. $(3: 829)$

Par ailleurs, les parents mentionnent les ressources humaines qui leur ont été offertes et la façon dont celles-ci se sont impliquées. La plupart des parents se souviennent plus particulièrement d'une personne-ressource, qui leur a apporté le soutien dont ils avaient besoin. Ils apprécient le soutien apporté par un « intervenant pivot » en qui ils ont confiance, qui leur fournit des conseils, des renseignements et qui les dirige vers d'autres ressources. Certains parents n'ont pas reçu l'appui d'une personne-ressource, et ils le précisent quand ils parlent des améliorations à apporter dans l'aide et les services offerts. Ils expliquent avoir eu besoin de beaucoup d'informations (guide, dépliant, personne-ressource), parce que le choc qu'ils vivent à la suite du diagnostic de l'enfant les empêche de bien comprendre toutes les explications fournies par les spécialistes.

En ce qui concerne les ressources techniques et matérielles (fauteuil roulant, poussette à grosses roues, adaptation du bain, vélo adapté, réveille-matin vibrant, ordinateur adapté, rampe d'accès, etc.), elles semblent faciliter grandement l'adaptation des enfants et augmenter leur qualité de vie. Certains parents semblent ainsi très satisfaits des ressources techniques et matérielles qu'ils ont reçues, alors que d'autres ont éprouvé des difficultés à les obtenir, soit en raison de longs délais d'attente ou de coûts trop élevés.

En matière de loisirs, certains centres ou associations offrent des services adaptés à l'incapacité de l'enfant. En fait, les associations organisent souvent des activités durant l'année, surtout lorsque les enfants sont en vacances. Plusieurs parents ont d'ailleurs mentionné l'importance de maintenir l'offre de ces activités :

II y a aussi la fondation X. Ça, c'est les activités, c'est au niveau des loisirs, c'est excellent, c'est numéro un, la fondation. II faudrait pas qu'on enlève ça, ils s'amusent, il $y$ a des gens, des amis qui ont des problèmes visuels, donc il ne se sent pas tout seul là-dedans, ils sont très bien encadrés, ils font toutes sortes d'activités. $(1: 2647)$ 
Certains parents déplorent toutefois qu'il n'y ait pas davantage d'activités organisées pour leur enfant. Quant aux parents sur le marché du travail, ils mentionnent que les journées pédagogiques posent problème.

Les parents parlent également des moyens de transport utilisés pour les déplacements. Certains services ne sont pas offerts dans toutes les villes. Ainsi, des parents utilisent parfois les transports adaptés lorsque c'est possible, sinon ils ont recours à d'autres moyens de transport :

C'est des transports adaptés, ça, c'est difficile. [...] c'est compliqué. Je ne suis pas capable d'avoir de services à moitié. Parce que je suis comme dans la zone tampon dans le fond. [...] (4:1497)

Les parents ont fait des commentaires positifs sur l'aide et les services reçus, en plus de mentionner des effets positifs de l'incapacité sur la vie familiale. Les parents apprécient notamment les efforts que certains déploient pour les aider :

[...] on leur demande quelque chose, ils se renseignent toujours, ils vont essayer de trouver qui va pouvoir t'aider et tout ça. Le centre de réadaptation, c'est très important. $(1: 1268)$

Les parents disent ne pas connaître suffisamment les services offerts pour leur enfant, et ils sont nombreux à déplorer que les services de santé (médecin spécialiste ou membre du personnel infirmier) ne fournissent pas de trousse d'information sur les différentes incapacités. Ils souhaiteraient y trouver une liste des organismes gouvernementaux et communautaires, des fondations, des regroupements, des instituts, des centres de réadaptation avec leurs coordonnées respectives et le type de services offerts.

[...] c'est qu'au départ, on n'avait nulle part où aller, on avait, après le diagnostic, on savait pas, absolument pas, à qui parler, on savait pas quoi faire, on s'en va où. $(6: 1775)$

Cette recherche de solutions semble plutôt difficile. De plus, elle affecte la vie familiale et exige des parents une grande implication. Comme nous l'avons mentionné plus haut, les parents qui ont accès à des personnes-ressources trouvent plus rapidement l'aide et les services dont ils ont besoin.

Des parents mentionnent les effets de l'incapacité de leur enfant sur leur situation financière. Ils doivent effectuer plusieurs achats supplémentaires en plus de voir leur revenu diminuer à la suite de choix de vie. Certains parents ont recours à des services privés pour combler le manque d'accès aux services publics ou les longs délais s'y rattachant, tout en regrettant les coûts élevés :

Très cher, mais tu as un résultat. Quand tu payes, tu peux réclamer et quand tu ne payes pas, tu dois accepter ce qu'ils te disent. C'est ça que j'ai remarqué quand j'allais au centre X. $(2: 315)$ 
Toutefois, d'autres parents ne peuvent offrir à leur enfant les services dont celui-ci aurait besoin :

J'ai pas les ressources financières pour aller chercher les services dont j'ai de besoin et le système de santé ne les trouve pas, les services. $(2: 1802)$

\section{Thème : Expérience familiale au quotidien}

Les commentaires des parents ont été regroupés en trois catégories : implication des parents et adaptation de la vie familiale, attitudes des autres à l'égard de la situation et difficultés dans la vie familiale. Les catégories sont présentées par ordre d'importance.

La catégorie «Implication des parents et adaptation de la vie familiale » regroupe les commentaires portant sur la très grande implication des parents d'enfants ayant des incapacités. Souvent, les deux parents se partagent les tâches, mais il arrive qu'un seul parent prenne la charge entière de l'enfant parce qu'il n'a pas de conjoint ou encore parce que l'autre parent ne s'engage pas. Certaines tâches sont divisées en fonction du temps. Par exemple, une mère qui ne travaille pas à l'extérieur de la maison s'occupe durant la semaine de son enfant ayant des incapacités et le père prend la relève la fin de semaine. Pour d'autres parents, ce sont les types de soins qui guident le partage des tâches.

C'est des trucs qu'on prend. Depuis que je m'en occupe, j'ai même changé de manière puis d'horaire. Parce qu'elle a grandi. [...] C'est sûr que des semaines qu'il travaille (le père), puisqu'il est pas là de la semaine, je dors la fin de semaine qui arrive. La fin de semaine, je suis vidée. Je repose mon dos, puis c'est lui qui s'en occupe. $(4: 1248)$

Peu importe la situation, le ou les parents doivent consacrer énormément de temps et d'énergie à leur enfant. En fait, ils considèrent qu'ils sont engagés auprès de lui en tout temps. Certains parents ajoutent que cette situation affecte leur carrière :

Et encore là combien d'énergie, c'est terrible toute l'énergie, la preuve: je ne suis même plus sur le marché du travail, je travaille à temps plein pour mon gars, à quelque part parce que c'est difficile, j'ai des tonnes de retours d'appels. $(2: 1885)$

Pour offrir une bonne qualité de vie à leur enfant et pour que celui-ci obtienne les meilleurs soins, les parents doivent développer un grand sens de l'organisation et apprendre à gérer les différents rendez-vous et les tâches quotidiennes :

[...] Les premières grandes difficultés que j'ai rencontrées, c'est d'être capable de gérer un horaire de travail comme professionnel et un horaire de rendez-vous médicaux qui pouvaient se déclencher à n'importe quelle heure. $(6: 19)$

[...] Comme parent, quand tu te ramasses un matin et puis que tu ne vas pas bien parce que tu es très grippée, que tu as une pneumonie, tu rentres travailler parce que tu n'as plus de congés, parce que tu les as passés pour ton enfant. $(6: 78)$ 
Certains employeurs se montrent toutefois assez compréhensifs :

Oui, il a fallu que je négocie une entente. Un moment donné, j'ai épuisé mes journées de maladie deux ans à l'avance, parce que je peux emprunter sur les années suivantes, selon les périodes de maladie. Donc, un moment donné, j'étais en dû de deux ans à l'avance sur les maladies au niveau du boulot, mais l'employeur ne me mettait pas de bâtons dans les roues, il était capable de comprendre cette situation-là. $(6: 282)$

Certains parents sont obligés d'abandonner leur travail ou choisissent de ne pas travailler pour se consacrer entièrement à leur enfant. S'occuper d'un enfant ayant des incapacités est un emploi à temps plein, ce qui a pour conséquence que les parents n'ont pas beaucoup de disponibilité ou qu'ils sont tout simplement trop fatigués pour retourner au travail :

[...] mais à cause de ça, c'est sûr qu'on lui donne beaucoup de temps, je ne travaille pas, je reste à la maison. Peut-être si elle n'avait pas eu de handicap, je travaillerais peut-être maintenant [...]. (1:760)

Au cours des entrevues, les parents décrivent les attitudes des autres personnes de leur entourage à l'égard de la situation. Ils mentionnent, notamment, que leurs relations familiales et amicales se sont peu à peu modifiées :

Mes amis sont disparus parce que mon garçon était autiste [rires], tu comprends. C'est comme ça et tu te refais de nouveaux amis, c'est ça. Une famille handicapée qui peut comprendre, ceux qui comprennent dans un sens, c'est qu'ils connaissent la maladie... $(2: 474)$

Parfois, les liens avec leur famille proche changent. La façon de réagir à la situation varie d'un membre de la famille à l'autre.

Oui, c'est plus, c'est plus profond comme relation, ça. Ben, faut voir le p'tit bonhomme pour comprendre. II a un charisme, il rentre à quelque part, puis il capte l'attention à tout le monde. [...] J'ai un de ses oncles un moment donné y dit [...] à c't'heure qu'on connaît ça là, si on l'aurait pas il nous manquerait quelque chose. $(7: 3085)$

Considérant les effets de l'incapacité sur leur carrière et sur leur vie quotidienne, les parents semblent vivre des moments difficiles. Les parents, plutôt que de planifier l'avenir, vivent dans le présent, sont en « mode de survie ». Ils rapportent ne pas avoir l'impression de contrôle sur leur vie, surtout dans les premiers temps suivant l'annonce du diagnostic. La santé peut également être affectée; les parents développent parfois des problèmes physiques à cause des préoccupations liées aux soins de leur enfant et ils manifestent de l'inquiétude au sujet de l'avenir de leur enfant :

[...] il y a des hommes et des femmes qui ont des enfants handicapés qui développent ça, la peur de mourir, qu'est-ce qui va arriver à l'enfant, ça, on développe ça. (2:530)

Donc, ça a tout un impact solide sur ta propre vie, ta propre santé. (6: 301) 


\section{DISCUSSION}

Le but de cette étude était de donner la parole aux parents afin qu'ils partagent leurs perceptions des effets de l'incapacité sur leur parcours de vie, leurs expériences d'utilisation des services et leurs besoins. II apparaît pertinent de dresser un portrait général des recommandations apportées par les parents ainsi que de celles qui sont davantage propres à certaines incapacités.

Plusieurs améliorations suggérées concernent les services offerts par les différents réseaux de services. Bien que de nombreux commentaires témoignent du fait que l'aide apportée par les services et les intervenants a pu jouer un rôle dans le processus d'adaptation, des suggestions sont formulées. II est souhaité, de la part des intervenants, qu'ils fassent preuve d'une plus grande sensibilité et qu'ils coordonnent mieux leurs interventions. Les parents souhaitent également une diminution du roulement de personnel et l'utilisation d'un plan de service commun à tous les intervenants.

Globalement, les services devraient être mieux pensés pour agir à certains moments cruciaux du processus, par exemple à l'annonce du diagnostic et dans les situations d'urgence. Certains types de services gagneraient à être développés. Ainsi, l'importance du soutien et de l'accompagnement psychologique est reconnue pour l'enfant et ses parents, mais également pour l'ensemble de la famille. II faudrait également améliorer les conditions facilitant l'adaptation, comme la possibilité de bénéficier d'un congé parental lors d'évènements tragiques ou de moments particulièrement importants. Pour les services déjà en place, une plus grande accessibilité, des délais d'attente moins grands et des suivis plus réguliers sont à privilégier. Une autre recommandation cible l'augmentation de services spécialisés, tels que l'orthophonie, la psychoéducation et l'orthopédagogie gestuelle, mais également celle des services contribuant à améliorer la qualité de vie, comme des accompagnateurs mieux formés pour les soirs et les fins de semaine, des services de garde lors des journées pédagogiques ou, encore, plus de ressources financières afin de pouvoir mieux organiser les transports, les loisirs et le répit.

Sur le plan de la sensibilisation, des suggestions sont faites afin d'offrir davantage d'information à la communauté. Il est alors question de renseignements généraux sur les différents types d'incapacités, mais également d'adaptations et d'interventions spécifiques, comme l'adaptation des lieux et des cours de LSQ dans les écoles ordinaires.

Certaines recommandations touchent des clientèles particulières. Ainsi, des parents d'enfants ayant des incapacités associées au traumatisme craniocérébral déplorent le manque d'appartements supervisés; les parents d'enfants ayant des incapacités motrices souhaitent une diminution du délai d'attente pour l'obtention de fauteuils roulants; les parents d'enfants ayant des incapacités visuelles souhaitent qu'il soit possible d'avoir un portable et un ordinateur de table, tandis que des parents d'enfants ayant des incapacités auditives souhaitent le développement de services d'interprétariat et une reconnaissance de la langue des signes québécoise. 
Le rôle des autres parents est grandement reconnu. Les parents rencontrés souhaiteraient un meilleur financement des milieux associatifs, de même que l'inclusion d'autres parents dans l'équipe de professionnels.

\section{CONCLUSION}

Une certaine prudence est de mise dans l'interprétation de ces résultats. II s'agit d'une étude qualitative menée auprès d'un petit nombre de personnes dont les profils sont fort variés. Les propos des parents rencontrés ne peuvent être considérés comme représentatifs de la réalité de l'ensemble des familles dont un membre présente des incapacités. Par ailleurs, l'adaptation des familles à la présence de l'incapacité présentée par un de ses membres est un processus complexe impliquant de multiples variables dont certaines ont d'ailleurs été documentées (notamment la situation économique). Bien que de nombreuses pistes d'action aient été dégagées à la suite de la rencontre de ces familles, il va sans dire que la recherche des déterminants de cette adaptation doit être poursuivie. À la lumière des parcours de vie des parents rencontrés, les familles témoignent posséder les compétences pour déterminer ce qui va « faire la différence » dans leur vie personnelle et familiale.

Rappelons, enfin, qu'une meilleure connaissance de la réalité des familles dont un membre est handicapé ne pourrait être possible sans que l'on s'attarde aux caractéristiques, aux défis et aux espoirs que ces familles partagent avec toutes les autres familles.

\section{RÉFÉRENCES BIBLIOGRAPHIQUES}

BogdAN, R.C., et S.K. BIKLEN (1998). Qualitative Research for Education: An Introduction to Theory and Methods, $3^{\mathrm{e}}$ édition, Boston, Allyn and Bacon.

CAmIRAND, J., et autres (2001). Enquête québécoise sur les limitations d'activités 1998, Québec, Institut de la statistique du Québec.

CAmiRAND, J., et J. AuBIN (2004). L'incapacité dans les familles québécoises: composition et conditions de vie des familles, santé et bien-être des proches, Québec, Institut de la statistique du Québec.

Crnic, K.A., W.N. Friedrich et M.T. GreEnBERg (1983). « Adaptation of families with mentallyretarded children: A model of stress, coping and family ecology », American Journal of Mental Deficiency, vol. 88, $\mathrm{n}^{\circ}$ 2, p. 125-138.

Cohen, L., L. MANion et K. Morrison (2000). Research Methods in Education, $5^{\mathrm{e}}$ édition, New York, Routledge. 
DARLING, R.B. (1991). « Parent-professional intervention: The roots of misunderstanding », dans M. Seligman (dir.), The Family with a Handicapped Child, New York, Grune and Stratton, p. 119-149.

Dionne, C., et N. Rousseau (2006). Évaluation qualitative de la situation des familles où vit une personne handicapée, Drummondville, Office des personnes handicapées du Québec.

DYson, L. (1993). «Response to the presence of a child with disabilities: Parental stress and family functioning over time », American Journal on Mental Retardation, vol. 98, p. 207-218.

GAGNIER, J.-P., et C. Gosselin (1999). «À la rencontre de grands-parents de petits-enfants ayant une déficience intellectuelle », Revue francophone de la déficience intellectuelle, vol. $8, n^{0} 1$, p. 55-64.

Gazzoni, P. (1998). L'intégration en garderie des enfants déficients : les attitudes, les connaissances et les conditions dans les milieux, Mémoire de maîtrise inédit, Université de Sherbrooke.

GURALNICK, M.J. (2000). «Early childhood intervention: Evolution of a system », dans M. WeHMEYER et J.R. PATTON (dir.), Mental Retardation in the 21st Century, Texas, Pro-Ed, p. 37-58.

HASTINGS, R.P., et A. BECK (2004). «Practitioner review: Stress intervention for parents of children with intellectual disabilities », Journal of Child Psychology and Psychiatry, vol. 45, $\mathrm{n}^{0} 8$, p. 1338-1349.

L'ÉCUYER, R. (1987). «L'analyse de contenu: notion et étapes », dans J.-P. DESLAURIERS, Les méthodes de la recherche qualitative, Sainte-Foy, Presses de l'Université du Québec, p. 49-65.

L'INSTITUT ROEHER INSTITUTE (2000). Beyond the Limits: Mothers Caring for Children with Disabilities, North York, Ontario, L'Institut Roeher Institute.

MuHR, T. (1997). ATLAS.ti. Scientific Software Development. Text Interpretation, Text Management and Theory Building [logiciel], Berlin.

Pelchat, D., et H. LefebVRe (2003). « Appropriation des savoirs parentaux dans la continuité des services pour les familles ayant un enfant atteint d'une déficience motrice cérébrale », Éducation et francophonie, vol. XXI, $\mathrm{n}^{\circ} 1$, printemps 2003.

Perreault, K. (1997). Pour mieux comprendre la différence. Une étude sur les besoins des personnes ayant une déficience intellectuelle et sur ceux de leurs proches, gouvernement du Québec, ministère de la Santé et des Services sociaux.

SCELLES, R. (1994). « La fratrie des personnes handicapées dans la littérature anglaise », Handicap et inadaptations - Les cahiers du CTNERHI, $\mathrm{n}^{\circ}$ 64, octobre-décembre, p. 73-89.

TÉTREAULT, S. (1993). «Étude exploratoire de la réalité des mères d'enfants ayant une incapacité motrice et des facteurs associés à la surcharge perçue », Sainte-Foy, Laboratoire de recherche, École de service social, Université Laval (Résultats de recherche).

Tétreault, S., J. Beaulieu, J. Bédard, G. Martin et V. Béguet (2002). « Handicap et rôles parentaux: perception des parents », dans S. TÉTREAULT (dir.), Famille et situation de handicap. Comprendre pour mieux intervenir, Sherbrooke, Éditions du CRP, p. 51-73 (Intégration sociale). 
Tougas, J. (2002). La restructuration des services éducatifs et de garde à l'enfance au Québec: les cinq premières années, Toronto, University of Toronto, Childcare Resource and Research Unit.

Trute, B., et D. Hiebert-Murphy (2002). « Family adjustment to childhood developmental disability: A measure of parent appraisal of family impacts », Journal of Pediatric Psychology, vol. 27, $\mathrm{n}^{0}$ 3, p. 271-280. 